\title{
BISMUT TYPE DIFFERENTIATION OF SEMIGROUPS
}

MARC ARNAUDON

Institut de Recherche Mathématique Avancée, Université Louis Pasteur, 7, rue René Descartes, F-67084 Strasbourg Cedex, France

ANTON THALMAIER

Institut für Angewandte Mathematik, Universität Bonn, Wegelerstraße 6, D-53115 Bonn, Germany

\section{ABSTRACT}

We present a unified approach to Bismut type differentiation formulas for heat semigroups on functions and forms. Both elliptic and hypoelliptic situations are considered. Nonlinear extensions apply to the case of harmonic maps between Riemannian manifolds and solutions to the nonlinear heat equation.

\section{INTRODUCTION}

Let $M$ be a smooth $n$-dimensional manifold. On $M$ consider a Stratonovich SDE with smooth coefficients of the type

$$
\delta X=A(X) \delta Z+A_{0}(X) \mathrm{d} t,
$$

where $A_{0} \in \Gamma(T M)$ is a vector field and $A: M \times \mathbb{R}^{r} \rightarrow T M,(x, z) \mapsto A(x) z$ a bundle map over $M$ for some $r$. The driving process $Z$ is assumed to be an $\mathbb{R}^{r}$-valued Brownian motion on some filtered probability space satisfying the usual completeness conditions. We write $X$. $(x)$ for the solution to (1.1) starting from the point $x \in M$, and denote its maximal lifetime by $\zeta(x)$.

Solutions to (1.1) are diffusions with generator given in Hörmander form as

$$
L=A_{0}+\frac{1}{2} \sum_{i=1}^{r} A_{i}^{2}
$$

with $A_{i}=A(\cdot) e_{i} \in \Gamma(T M)$ for $i=1, \ldots, r$. We consider the minimal semigroup to $(1.1)$

$$
\left(P_{t} f\right)(x)=\mathbf{E}\left[\left(f \circ X_{t}(x)\right) \mathbb{I}_{\{t<\zeta(x)\}}\right],
$$

likewise let

$$
u(x)=\mathbf{E}\left[f \circ X_{\tau(x)}(x)\right]
$$


be the representation of an $L$-harmonic function $u$ on some domain $D \subset M$, where $\tau(x)$ is the first exit time of $X$ from $D$ when starting at $x$.

Our aim, if possible, is to find formulas for the differential $\mathrm{d}\left(P_{t} f\right)$, respectively $\mathrm{d} u$, which do not involve derivatives of $f$. Such differentiation formulas are sometimes called "Bismut type" formulas. For a survey on applications of differentiation formulas we refer to (Elworthy and $\mathrm{Li}, 1994)$.

\section{AN INTEGRATION BY PARTS ARGUMENT}

In this section we explain a general integration by parts argument at the level of local martingales which will serve as a key ingredient in our approach to differentiation formulas. Suppose that we are given a time-space function $F$ transforming our diffusion into a continuous local martingale $F(\cdot, X .(x))$. All processes considered in this paper are supposed to have continuous paths. The function $F$ is assumed to be $C^{1}$ in the space variable with a derivative continuous in both variables. We have mainly two cases in mind, namely

(1) $F\left(\cdot, X_{\bullet}(x)\right)=u(X .(x))$, where $u$ is some $L$-harmonic function, and

(2) $F(\cdot, X .(x))=\left(P_{t-\bullet} f\right)(X .(x))$ for some $f \in B(M)$, i.e. $f$ is a bounded measurable function on $M$.

Suppose further that $W_{0, s}: T_{x} M \rightarrow T_{X_{s}(x)} M$ are linear transports on $M$ along $X(x)$ such that, for each $v \in T_{x} M$,

$$
\mathrm{d} F(s, \cdot)_{X_{s}(x)} W_{0, s} v, \quad s \geqslant 0,
$$

is a local martingale. We assume that $W_{s, 0}=W_{0, s}^{-1}$ and $W_{0,0}=\operatorname{id}_{T_{x} M}$. Note that a possible choice for $W_{0, s}$ is for instance $W_{0, s}=T_{x} X_{s}$. This follows from the fact that the derivative of a $C^{1}$-family (w.r.t. compact convergence in probability) of local martingales is again a local martingale (Arnaudon and Thalmaier, 1998a).

Lemma 1. For $F(\cdot, X .(x))$ as above, let $W_{0, .}$ be a linear transport along $X(x)$ such that (2.1) are local martingales. Then, for any $\mathbb{R}^{r}$-valued process $k$ in $L_{\mathrm{loc}}^{2}(Z)$ and any $v \in T_{x} M$,

$$
\begin{aligned}
N_{s}:= & \mathrm{d} F(s, \cdot)_{X_{s}(x)} W_{0, s}\left(v-\int_{0}^{s} W_{0, r}^{-1} A\left(X_{r}(x)\right) k_{r} \mathrm{~d} r\right) \\
& +F\left(s, X_{s}(x)\right) \int_{0}^{s}\langle k, \mathrm{~d} Z\rangle
\end{aligned}
$$

is a local martingale for $0 \leqslant s<\sigma$, where $[0, \sigma[$ denotes the stochastic interval on which $F\left(\cdot, X_{\bullet}(x)\right)$ is defined, e.g. $\sigma=t \wedge \zeta(x)$ or $\tau(x)$, etc. 
Proof. We have to show that

$$
\mathrm{d} F(s, \cdot)_{X_{s}(x)} W_{0, s} \int_{0}^{s} W_{0, r}^{-1} A\left(X_{r}(x)\right) k_{r} \mathrm{~d} r-F\left(s, X_{s}(x)\right) \int_{0}^{s}\langle k, \mathrm{~d} Z\rangle, \quad s \geqslant 0,
$$

is a local martingale. Let $h_{s}=\int_{0}^{s} W_{0, r}^{-1} A\left(X_{r}(x)\right) k_{r} \mathrm{~d} r$. Then $\mathrm{d} F(s, \cdot)_{X_{s}(x)} W_{0, s} h_{s}-$ $\int_{0}^{s} \mathrm{~d} F(r, \cdot)_{X_{r}(x)} W_{0, r} \mathrm{~d} h_{r}, s \geqslant 0$, is a local martingale, and the claim follows upon noting that $F\left(s, X_{S}(x)\right)=F(0, x)+\int_{0}^{s} \mathrm{~d} F(r, \cdot)_{X_{r}(x)} A\left(X_{r}(x)\right) \mathrm{d} Z_{r}$ which implies that $\int_{0}^{s} \mathrm{~d} F(r, \cdot)_{X_{r}(x)} W_{0, r} \mathrm{~d} h_{r}-F\left(s, X_{s}(x)\right) \int_{0}^{s}\langle k, \mathrm{~d} Z\rangle, s \geqslant 0$, is a local martingale.

The procedure is now straightforward. Suppose that we are able to choose the process $k$ in (2.2) in such a way that the following two conditions hold:

(a) $\int_{0}^{\sigma} W_{0, r}^{-1} A\left(X_{r}(x)\right) k_{r} \mathrm{~d} r=v$ a.s., and

(b) $N^{\sigma} \equiv\left(N_{t \wedge \sigma}\right)_{t \geqslant 0}$ is a uniformly integrable martingale.

Then, evaluating $\mathbf{E}\left[N_{0}\right]=\mathbf{E}\left[N_{\sigma}\right]$ gives a formula for $\mathrm{d} F(0, \cdot)_{x} v$ in terms of the process $F\left(\cdot, X_{.}(x)\right)$. In the elliptic case, both (a) and (b) can easily be achieved (Thalmaier, 1997), by exploiting the fact that there is a right-inverse to the process $A(X .(x))$.

\section{THE ELLIPTIC CASE}

Suppose that our system (1.1) is elliptic, i.e. $A(x): \mathbb{R}^{r} \rightarrow T_{x} M$ is onto for each $x \in$ $M$. Then there is a Riemannian metric $g$ on $M$ which makes $A(x)^{*}: T_{x} M \rightarrow \mathbb{R}^{r}$ to an isometric inclusion for each $x \in M$. In particular, the generator (1.2) then writes as

$$
L=\frac{1}{2} \Delta_{M}+V
$$

where $\Delta_{M}$ is the Laplace-Beltrami operator on $M$ with respect to the metric $g$ and $V$ is a first order term, i.e. $V \in \Gamma(T M)$.

In this case, there is an intrinsic choice for the linear transports $W_{0,}$ in (2.1). To this end, let $W_{0, s}: T_{x} M \rightarrow T_{X_{s}(x)} M$ be defined by the following covariant equation along $X(x)$ :

$$
\frac{D}{\mathrm{~d} s} W_{0, s}=-\frac{1}{2} \operatorname{Ric}^{M}\left(W_{0, s}, \cdot\right)^{\#}+\nabla V\left(W_{0, s}\right), \quad W_{0,0}=\mathrm{id}_{T_{x} M} .
$$

Then the local martingale property of (2.1) is elementary to check: indeed, this can be done either directly by applying Weitzenböck's formula to the Laplacian on 1forms (see, for instance, Thalmaier, 1998), or by using the method of filtering out redundant noise from the derivative process $T_{x} X$ (cf. Elworthy and Yor, 1993). Let

$$
B_{r}=\int_{0}^{r} / /_{0, s}^{-1} A\left(X_{s}(x)\right) \mathrm{d} Z_{s}
$$


be the martingale part of the anti-development $\mathcal{A}(X):=\int_{0}^{\bullet} / /_{0, s}^{-1} \delta X_{S}$ of $X=X(x)$. Obviously, $B$ is a Brownian motion on $T_{x} M$ and, by definition, $A\left(X_{s}(x)\right) \mathrm{d} Z_{s}=$ $/ /_{0, s} \mathrm{~d} B_{s}$. In this situation, Lemma 1 is easily adapted to give the local martingale property of

$$
N_{s}=\mathrm{d} F(s, \cdot)_{X_{s}(x)} W_{0, s}\left(v-\int_{0}^{s} W_{0, r}^{-1} / /_{0, r} k_{r} \mathrm{~d} r\right)+F\left(s, X_{s}(x)\right) \int_{0}^{s}\langle k, \mathrm{~d} B\rangle .
$$

Taking $h_{s}=v-\int_{0}^{s} W_{0, r}^{-1} / /_{0, r} k_{r} \mathrm{~d} r$, we get the following typical applications in the elliptic case, see (Thalmaier, 1997) for details. In the sequel, let $\mathbb{H}\left(\mathbb{R}_{+}, T_{x} M\right)$ denote the Cameron-Martin space of paths $\gamma: \mathbb{R}_{+} \rightarrow T_{x} M$ of finite energy.

THEOREM 2 (Differentiation formula for heat semigroups). Let $f: M \rightarrow \mathbb{R}$ be bounded measurable, $x \in M$ and $v \in T_{x} M$. Then, for any bounded adapted process $h$ with paths in $\mathbb{H}\left(\mathbb{R}_{+}, T_{x} M\right)$ such that $\left(\int_{0}^{\tau(x) \wedge t}\left|\dot{h}_{S}\right|^{2} \mathrm{~d} s\right)^{1 / 2} \in L^{1}$, and the property that $h_{0}=v, h_{s}=0$ for all $s \geqslant \tau(x) \wedge t$, the following formula holds:

$$
\mathrm{d}\left(P_{t} f\right)_{x} v=-\mathbf{E}\left[f\left(X_{t}(x)\right) 1_{\{t<\zeta(x)\}} \int_{0}^{\tau(x) \wedge t}\left\langle W_{0, s}\left(\dot{h}_{s}\right), / /_{0, s} \mathrm{~d} B_{s}\right\rangle\right],
$$

where $\tau(x)$ is the first exit time of $X(x)$ from some relatively compact open neighbourhood D of $x$.

THEOREM 3 (Differentiation formula for harmonic functions). Let $D \subset M$ be $a$ nonvoid relatively compact open subset with smooth boundary $\partial D \neq \mathrm{ffl}$, and $\tau(x)=$ $\inf \left\{t \geqslant 0: X_{t}(x) \notin D\right\}$ the first exit time of $X$ from $D$ when started at $x \in D$. Let $u \in C(\bar{D})$ be L-harmonic on $D$. Then

$$
(\mathrm{d} u)_{x} v=-\mathbf{E}\left[u\left(X_{\tau(x)}(x)\right) \int_{0}^{\tau(x)}\left\langle W_{s}\left(\dot{h}_{s}\right), / /_{0, s} \mathrm{~d} B_{s}\right\rangle\right]
$$

for any bounded adapted process $h$ with paths in $\mathbb{H}\left(\mathbb{R}_{+}, T_{x} M\right)$ such that $h_{0}=v$, $h_{s} \equiv 0$ for $s \geqslant \tau(x)$, and the property that $\left(\int_{0}^{\tau(x)}\left|\dot{h}_{s}\right|^{2} \mathrm{~d} s\right)^{1 / 2} \in L^{1+\varepsilon}$ for some $\varepsilon>0$.

For possible choices of the process $h$ see (Thalmaier and Wang, 1998) where formula (3.5) is used to prove local gradient estimates of harmonic functions.

\section{NONLINEAR GENERALIZATIONS}

The arguments of the previous section are easily extended to nonlinear situations, e.g. to harmonic maps $u: M \rightarrow N$ between Riemannian manifolds, and more generally, to solutions of the nonlinear heat equation

$$
\frac{\partial}{\partial t} u=\frac{1}{2} \Delta u
$$


for maps between $M$ and $N$, where $\Delta u=\operatorname{trace} \nabla \mathrm{d} u$ is the tension of $u$. In these cases

(1) $F_{s} \equiv F\left(s, X_{s}(x)\right)=u\left(X_{s}(x)\right)$, respectively

(2) $F_{s} \equiv F\left(s, X_{s}(x)\right)=u_{t-s}\left(X_{s}(x)\right)$,

define $\nabla$-martingales on $N$ for Brownian motions $X$ on $M$.

DEFINITION 4 (Arnaudon and Thalmaier, 1998b). For a continuous semimartingale $Y$ taking values in a manifold $N$, endowed with a torsionfree connection $\nabla$, the geodesic transport (also called deformed or damped transport; Dohrn-Guerra transport) $\Theta_{0, t}: T_{Y_{0}} N \rightarrow T_{Y_{t}} N$ on $N$ along $Y$ is defined by the following covariant equation along $Y$ :

$$
\mathrm{d}\left(/ / /_{0, \bullet}^{-1} \Theta_{0, .}\right)=-\frac{1}{2} / /_{0, .}^{-1} R\left(\Theta_{0, .}, \mathrm{d} Y\right) \mathrm{d} Y, \quad \Theta_{0,0}=\mathrm{id},
$$

where $/ /_{0, t}: T_{Y_{0}} N \rightarrow T_{Y_{t}} N$ is parallel transport on $N$ along $Y$ and $R$ denotes the curvature tensor to $\nabla$. The $T_{Y_{0}} N$-valued process

$$
\mathcal{A}_{\text {def }}(Y)=\int_{0}^{\infty} \Theta_{0, s}^{-1} \delta Y_{s}
$$

is called deformed anti-development of $Y$.

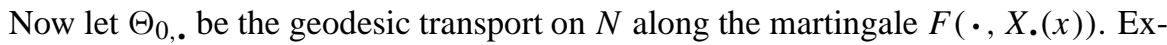
ploiting the fact that the Stratonovich integral in (4.3) can be replaced by an Itô integral, we get

$$
\mathcal{A}_{\text {def }}(F(\cdot, X .(x)))=\int_{0}^{\infty} \Theta_{0, s}^{-1} \mathrm{~d} F(s, \cdot)_{X_{s}(x)} A\left(X_{s}(x)\right) \mathrm{d} Z_{s} .
$$

Further, by taking into account that (Arnaudon and Thalmaier, 1998b)

$$
m_{s}:=\Theta_{0, s}^{-1} \mathrm{~d} F(s, \cdot)_{X_{s}(x)} W_{0, s}
$$

is local martingale in $T_{x}^{*} M \otimes T_{F(0, x)} N$, we obtain the following generalization of Lemma 1 to the nonlinear case.

LemMA 5. Let $F(\cdot, X .(x)), x \in M$, be a family of $\nabla$-martingales on $N$, as above. Then, for any predictable $\mathbb{R}^{r}$-valued process $k$ in $L_{\mathrm{loc}}^{2}(Z)$,

$$
\Theta_{0, \bullet}^{-1} \mathrm{~d} F(\cdot, X .(x)) W_{0, .} \int_{0} W_{0, s}^{-1} / /_{0, s} k_{s} \mathrm{~d} s-\mathcal{A}_{\mathrm{def}}(F(\cdot, X .(x))) \int_{0}\langle k, \mathrm{~d} Z\rangle
$$

is a local martingale in $T_{F(0, x)} N$.

Following the lines of Section 3, Lemma 5 leads to explicit differentiation formulas in the nonlinear case, cf. (Arnaudon and Thalmaier, 1998b) for technical details. 
THEOREM 6 (A nonlinear differentiation formula). Let $u:[0, t] \times M \rightarrow N$ be $a$ smooth solution of equation (4.1) and $v \in T_{x} M$. Then

$$
\left(\mathrm{d} u_{t}\right)_{x} v=-\mathbf{E}\left[\mathcal{A}_{\operatorname{def}}\left(u\left(t-\cdot, X_{.}(x)\right)\right)_{\tau(x) \wedge t} \int_{0}^{\tau(x) \wedge t}\left\langle W_{0, s} \dot{h}_{s}, / /_{0, s} \mathrm{~d} B_{s}\right\rangle\right]
$$

for any bounded adapted process $h$ with sample paths in the Cameron-Martin space $\mathbb{H}\left([0, t], T_{x} M\right)$ such that $\left(\int_{0}^{\tau(x) \wedge t}\left|\dot{h}_{s}\right|^{2} \mathrm{~d} s\right)^{1 / 2} \in L^{1+\varepsilon}$ for some $\varepsilon>0$, and the property that $h_{0}=v, h_{s}=0$ for all $s \geqslant \tau(x) \wedge t$; here $\tau(x)$ is again the first exit time of $X(x)$ from some relatively compact neighbourhood $D$ of $x$.

In the same way, formulas for the differential of harmonic maps $u: M \rightarrow N$ between Riemannian manifolds can be derived,

$$
(\mathrm{d} u)_{x} v=-\mathbf{E}\left[\mathcal{A}_{\operatorname{def}}(u(X .(x)))_{\tau(x)} \int_{0}^{\tau(x)}\left\langle W_{0, s} \dot{h}_{s}, / /_{0, s} \mathrm{~d} B_{s}\right\rangle\right],
$$

with assumptions analogous to Theorem 6. Note that the stochastic integral in (4.4) depends only on the local geometry of $M$ about the point $x$. Formula (4.4) has been used in (Arnaudon and Thalmaier, 1998b) to prove local gradient estimates for harmonic maps of bounded dilatation, recovering theorems of Goldberg-IshiharaPetridis and Shen as special cases.

\section{EXTENSIONS TO DIFFERENTIAL FORMS}

In this section we sketch extensions from functions to differential forms. For more general results in this direction, e.g. for sections in vector bundles, see (Driver and Thalmaier, 1999).

On a Riemannian manifold $M$, endowed with the Levi-Civita connection $\nabla$, consider the exterior algebra bundle

$$
E=\Lambda T^{*} M \equiv \bigoplus_{p \geqslant 0} \Lambda^{p} T^{*} M
$$

Let $\Delta$ denote the de Rham-Hodge Laplacian on $\Gamma(E)$ with sign convention

$$
\Delta=-\left(\mathrm{dd}^{*}+\mathrm{d}^{*} \mathrm{~d}\right),
$$

where $\mathrm{d}_{\text {and }} \mathrm{d}^{*}$ are the exterior differential, respectively codifferential. Note that for $a \in \Gamma(E)$,

$$
\mathrm{d} a=c^{+} \nabla a, \quad \mathrm{~d}^{*} a=-c^{-} \nabla a,
$$

where $\nabla: \Gamma(E) \rightarrow \Gamma\left(T^{*} M \otimes E\right)$ is the induced covariant derivative on $E$ and

$$
\begin{aligned}
& c^{+}=\wedge: T^{*} M \otimes \Lambda T^{*} M \rightarrow \Lambda T^{*} M, \quad s \otimes v \mapsto s \wedge v, \\
& c^{-}=\left\llcorner: T^{*} M \otimes \Lambda T^{*} M \rightarrow \Lambda T^{*} M, \quad s \otimes v \mapsto s\llcorner v\right.
\end{aligned}
$$


denote wedge product and contraction in $E=\Lambda T^{*} M$, respectively. On $\Lambda T M$ the operations $c^{ \pm}$are defined analogously. Recall that, by definition, $s\llcorner v=$ $\sum_{k=1}^{p}(-1)^{k+1}\left\langle s, v_{k}\right\rangle v_{1} \wedge \cdots \widehat{v}_{k} \wedge \cdots v_{p}$ for $v=v_{1} \wedge \cdots \wedge v_{p}$. Further, by Weitzenböck's formula,

$$
\Delta a=\square a-\mathcal{R} a,
$$

where $\square a=\operatorname{trace} \nabla^{2} a$ is the "rough Laplacian" on $\Gamma(E)$ and $\mathcal{R} \in \Gamma(\operatorname{Hom} E)$ the Weitzenböck curvature term.

For $x \in M$ fixed, the Weitzenböck term $\mathcal{R}$ can be used to define a process $Q$ with values in $\operatorname{Aut}\left(E_{x}\right)$ via the pathwise equation

$$
\frac{\mathrm{d}}{\mathrm{d} t} Q_{t}=-\frac{1}{2} Q_{t} \mathcal{R}_{/ / 0, t}, \quad Q_{0}=\mathrm{id}_{E_{x}},
$$

where $\mathcal{R}_{/ / 0, t}=/ /_{0, t}^{-1} \circ \mathcal{R}_{X_{t}(x)} \circ / /_{0, t}$.

For the remainder of this section, let $\langle\cdot, \cdot\rangle$ denote the natural pairing between $\Lambda T_{x}^{*} M$ and $\Lambda T_{x} M$, and let $Q_{t}^{*} \in \operatorname{Aut}\left(\Lambda T_{x} M\right)$ be determined by $\left\langle Q_{t} v, w\right\rangle=$ $\left\langle v, Q_{t}^{*} w\right\rangle$ for $v \in \Lambda T_{x}^{*} M, w \in \Lambda T_{x} M$.

The following lemma is crucial for derivative formulas in our situation, see (Driver and Thalmaier, 1999) for generalizations in various directions.

LEMMA 7. Let a be a solution to the heat equation on differential forms, i.e. on $\Gamma(E), \frac{\mathrm{d}}{\mathrm{d} t} a=\frac{1}{2} \Delta a$, where $\Delta$ is the de Rham-Hodge Laplacian given by (5.1). Then

$$
\begin{aligned}
N_{s}= & \left\langle Q_{s} / /_{0, s}^{-1} d a_{t-s}\left(X_{s}(x)\right), \ell(s)\right\rangle \\
& -\left\langle Q_{s} / /_{0, s}^{-1} a_{t-s}\left(X_{s}(x)\right), \int_{0}^{s}\left(Q_{r}^{*}\right)^{-1}\left(\mathrm{~d} B_{r}\left\llcorner Q_{r}^{*} \dot{\ell}(r)\right)\right\rangle\right.
\end{aligned}
$$

and

$$
\begin{aligned}
N_{s}^{*}= & \left\langle Q_{s} / /_{0, s}^{-1} d^{*} a_{t-s}\left(X_{s}(x)\right), \ell(s)\right\rangle \\
& +\left\langle Q_{s} / /_{0, s}^{-1} a_{t-s}\left(X_{s}(x)\right), \int_{0}^{s}\left(Q_{r}^{*}\right)^{-1}\left(\mathrm{~d} B_{r} \wedge Q_{r}^{*} \dot{\ell}(r)\right)\right\rangle
\end{aligned}
$$

are local martingales for any adapted process $\ell$ with paths in the Cameron-Martin space $\mathbb{H}\left([0, t], \Lambda T_{X} M\right)$. Here $X(x)$ is a Brownian motion on $M$ starting from $x$ and $B$ its anti-development taking values in $T_{x} M$.

The following theorem is a typical application of Lemma 7, see also (Elworthy and Li, 1998). For simplicity, we assume $M$ to be compact. For $L^{2}$-sections $a$ of $E$, let the semigroup $P_{t} a=\mathrm{e}^{t / 2 \bar{\Delta}} a$ be defined by means of the spectral theorem, where $\bar{\Delta}$ denotes the closure of $\Delta$. Then $\left(P_{t} a\right)_{x}=\mathbf{E}\left[Q_{t} / /_{0, t}^{-1} a\left(X_{t}(x)\right)\right]$. 
THEOREM 8. Let $M$ be a compact manifold, $a \in L^{2}-\Gamma\left(\Lambda T^{*} M\right)$ be a bounded $L^{2}$ section and $v \in \Lambda T_{x} M$ for some $x \in M$. Then

$$
\begin{aligned}
& \left(d P_{t} a\right)_{x} v=-\mathbf{E}\left[\left\langle Q_{t} / /_{0, t}^{-1} a\left(X_{t}(x)\right), \int_{0}^{t}\left(Q_{s}^{*}\right)^{-1}\left(\mathrm{~d} B_{s}\left\llcorner Q_{s}^{*} \dot{\ell}(s)\right)\right\rangle\right],\right. \\
& \left(d^{*} P_{t} a\right)_{x} v=\mathbf{E}\left[\left\langle Q_{t} / /_{t, a}^{-1}\left(X_{t}(x)\right), \int_{0}^{t}\left(Q_{s}^{*}\right)^{-1}\left(\mathrm{~d} B_{s} \wedge Q_{s}^{*} \dot{\ell}(s)\right)\right\rangle\right]
\end{aligned}
$$

for any adapted process $\ell$ with sample paths in $\mathbb{H}\left([0, t], \Lambda T_{x} M\right)$ such that $\ell(0)=v$, $\ell(t)=0$, and the property that $\left(\int_{0}^{t}|\dot{\ell}(s)|^{2} \mathrm{~d} s\right)^{1 / 2} \in L^{1}$.

\section{FORMULAS IN THE HYPOELLIPTIC CASE}

In the rest of this survey, we give a generalization of the results in Section 3. We consider again our system (1.1) but now with the assumption that the generator $L=$ $A_{0}+\frac{1}{2} \sum_{i=1}^{r} A_{i}^{2}$ is only hypoelliptic, i.e.

$$
\operatorname{Lie}\left(A_{i},\left[A_{0}, A_{i}\right]: i=1, \ldots, r\right)(x)=T_{x} M, \quad \text { for all } x \in M .
$$

In other words: the ideal in $\operatorname{Lie}\left(A_{0}, A_{1}, \ldots, A_{r}\right)$ generated by $\left(A_{1}, \ldots, A_{r}\right)$ is assumed to be full at each $x \in M$. We want to extend Theorem 1 and Theorem 2 to the hypoelliptic situation.

Under hypothesis (H1), the "Malliavin Covariance"

$$
C_{t}(x)=\sum_{i=1}^{r} \int_{0}^{t}\left(X_{s *}^{-1} A_{i}\right)_{x} \otimes\left(X_{s *}^{-1} A_{i}\right)_{x} \mathrm{~d} s
$$

defines a positive symmetric bilinear form on $T_{x}^{*} M$ for $x \in M$ such that $t<\zeta(x)$. We read $C_{t}(x): T_{x}^{*} M \rightarrow T_{x} M, C_{t}(x)^{-1}: T_{x} M \rightarrow T_{x}^{*} M$ and write

$$
C_{t}(x)=\int_{0}^{t}\left(X_{s *}^{-1} A\right)_{x}\left(X_{s *}^{-1} A\right)_{x}^{*} \mathrm{~d} s
$$

where $\left(X_{s *}^{-1} A\right)_{x}: \mathbb{R}^{r} \rightarrow T_{x} M$ is defined by $z \mapsto \sum_{i=1}^{r}\left(X_{s *}^{-1} A_{i}\right)_{x} z^{i}$. Recall that by Lemma 1 , for any $\mathbb{R}^{r}$-valued $k$ process in $L_{\text {loc }}^{2}(Z)$,

$$
N_{s}:=\mathrm{d} F(s, \cdot) X_{s *}\left[v-\int_{0}^{s} X_{r *}^{-1} A\left(X_{r}(x)\right) k_{r} \mathrm{~d} r\right]+F\left(s, X_{s}(x)\right) \int_{0}^{s}\langle k, \mathrm{~d} Z\rangle
$$

is a local martingale on $[0, t \wedge \zeta(x)[$.

Remark 9. For $x \in M$ and $v \in T_{x} M$ one may consider the system $\dot{h}_{s}=-\left(X_{s *}^{-1} A\right)_{x} k_{s}$, $h_{0}=v$. Note that if we are able to find a predictable $k$ with values in $\mathbb{R}^{r}$ such that

(1) $h_{\sigma}=0$ where $\sigma=\tau(x)$ or $\sigma=\tau(x) \wedge t$, and in addition, 
(2) $\left(\int_{0}^{\sigma}\left|k_{S}\right|^{2} \mathrm{~d} s\right)^{1 / 2} \in L^{1+\varepsilon}$ for some $\varepsilon>0$,

then the results of Section 3 in the elliptic case carry over almost verbatim.

However, it can be shown (Arnaudon and Thalmaier, 1998c) that, in general, this is not possible in the hypoelliptic situation. A counterexample in three dimensions (a two-dimensional Brownian motion and its Lévy area as a third coordinate) has been communicated to us by Jean Picard.

The following result in the hypoelliptic case is taken from (Arnaudon and Thalmaier, 1998c). A similar result can be given for $L$-harmonic functions.

THEOREM 10. Let $M$ be a smooth manifold and $f: M \rightarrow \mathbb{R}$ be bounded measurable. Assume that (H1) holds. Let $v \in T_{x} M$ and $t>0$. Then, for $P_{t} f$ defined by (1.3), there is a formula of the type

$$
\left(P_{t} f\right)_{x} v=\mathbf{E}\left[f\left(X_{t}(x)\right) \mathbb{I}_{\{t<\zeta(x)\}} \Phi_{t} v\right],
$$

where $\Phi_{t}$ is a $T_{x}^{*} M$-valued random variable, $L^{p}$-integrable for $1 \leqslant p<\infty$ and local in the following sense: for any relatively compact neighbourhood $D$ of $x$ in $M$ there is a choice for $\Phi_{t}$ which is $\mathcal{F}_{\sigma}$-measurable where $\sigma=t \wedge \tau(x)$ and $\tau(x)$ is the first exit time of $X$ from $D$ when starting at $x$.

We briefly sketch some arguments underlying Theorem 10. For simplicity, we assume that $M$ is compact and $f$ is $C^{1}$. Our sketch of proof does not show that $\Phi_{t}$ is indeed local: to see this, the given arguments have to be reformulated in terms of local martingales (cf. Arnaudon and Thalmaier, 1998c).

Let $a$ be a predictable process with values in $T_{x}^{*} M \otimes \mathbb{R}^{r}$ and $\lambda \in T_{x} M \equiv \mathbb{R}^{n}$ (locally about 0 ) such that for $t>0$,

$$
\mathbf{E}\left[\exp \left(\frac{1}{2} \int_{0}^{t}\left|a_{s} \lambda\right|^{2} \mathrm{~d} s\right)\right]<\infty .
$$

Let $\mathrm{d} Z^{\lambda}=\mathrm{d} Z+a \lambda \mathrm{d} t$ and consider the Girsanov exponential $G_{\text {. }}^{\lambda}$ defined by

$$
G_{t}^{\lambda}=\exp \left(-\int_{0}^{t}\langle a \lambda, \mathrm{d} Z\rangle-\frac{1}{2} \int_{0}^{t}|a \lambda|^{2} \mathrm{~d} s\right) .
$$

We write $X^{\lambda}$ for the flow to our SDE driven by the perturbed Brownian motion $Z^{\lambda}$, analogously $C_{.}^{\lambda}(x)$, etc. By Girsanov's theorem, we find that $H(\lambda)=\sum_{\ell} \mathbf{E}\left[f\left(X_{t}^{\lambda}(x)\right)\right.$. $\left.G_{t}^{\lambda} \cdot\left(C_{t}^{\lambda}(x)^{-1}\right)_{k \ell} v^{\ell}\right]$ is independent of $\lambda$ for each $k$. Thus $\left.\frac{\partial}{\partial \lambda_{k}}\right|_{\lambda=0} H(\lambda)=0$, from where we conclude that

$$
\sum_{i, k, \ell} \mathbf{E}\left[\left(D_{i} f\right)\left(X_{t}(x)\right)\left(X_{t *} \int_{0}^{t}\left(X_{s *}^{-1} A\right)_{x} a_{s} \mathrm{~d} s\right)_{i k}\left(C_{t}(x)^{-1}\right)_{k \ell} v^{\ell}\right]
$$




$$
=-\sum_{k, \ell} \mathbf{E}\left[\left.f\left(X_{t}(x)\right) \frac{\partial}{\partial \lambda_{k}}\right|_{\lambda=0}\left(G_{t}^{\lambda}\left(C_{t}^{\lambda}(x)^{-1}\right)_{k \ell} v^{\ell}\right)\right] .
$$

Recall that $\left(X_{s *}^{-1} A\right)_{x} \in \mathbb{R}^{r} \otimes T_{x} M$. Now the idea is to set $a_{s}=a_{s}^{n}=\left(X_{s *}^{-1} A\right)_{x}^{*} \mathbb{I}_{\left\{s \leqslant \tau_{n}\right\}} \in$ $T_{x}^{*} M \otimes \mathbb{R}^{r}$, where $\tau_{n} \nearrow t$ is a sequence of stopping times such that each $a^{n}$. satisfies the integrability condition. This gives $\mathbf{E}\left[(\mathrm{d} f)_{X_{t}(x)} X_{t *} C_{\tau_{n}}(x) C_{t}(x)^{-1} v\right]=$ $\mathbf{E}\left[\left(f \circ X_{t}(x)\right) \cdot \Phi_{t}^{n} v\right]$.

Finally, taking the limit as $n \rightarrow \infty$, we get $\mathrm{d}\left(P_{t} f\right)_{x} v=\mathbf{E}\left[(\mathrm{d} f)_{X_{t}(x)} X_{t *} v\right]=$ $\mathbf{E}\left[\left(f \circ X_{t}(x)\right) \cdot \Phi_{t} v\right]$, where

$$
\begin{aligned}
\Phi_{t} v= & \left(\int_{0}^{t}\left(X_{s *}^{-1} A\right)_{x} \mathrm{~d} Z_{s}\right) C_{t}^{-1}(x) v \\
& +\sum_{k, \ell}\left(C_{t}(x)^{-1}\left(\left.\frac{\partial}{\partial \lambda_{k}}\right|_{\lambda=0} C_{t}^{\lambda}(x)\right) C_{t}(x)^{-1}\right)_{k \ell} v^{\ell} .
\end{aligned}
$$

\section{REFERENCES}

Arnaudon, M. and Thalmaier, A. (1998a). Stability of stochastic differential equations in manifolds. In Séminaire de Probabilités XXXII, pp. 188-214. Lecture Notes in Math. 1686, Springer, Berlin.

Arnaudon, M. and Thalmaier, A. (1998b). Complete lifts of connections and stochastic Jacobi fields. J. Math. Pures Appl. 77, 283-315.

Arnaudon, M. and Thalmaier, A. (1998c). The differentiation of hypoelliptic diffusion semigroups. Preprint.

Driver, B. K. and Thalmaier, A. (1999). Heat kernel derivative formulas for vector bundles. Preprint.

Elworthy, K. D. and Li, X.-M. (1994). Differentiation of heat semigroups and applications. In: Probability Theory and Mathematical Statistics. Proc. 6th Vilnius Conf., pp. 239-251, B. Grigelionis et al. (Eds), VSP, Utrecht/TEV, Vilnius.

Elworthy, K. D. and Li, X.-M. (1998). Bismut type formulae for differential forms. C.R. Acad. Sci. Paris Sér. I Math. 327, 87-92.

Elworthy, K. D. and Yor, M. (1993) Conditional expectations for derivatives of certain stochastic flows. In: Séminaire de Probabilités XXVII, pp. 159-172, Lecture Notes in Math. 1557, Springer, Berlin.

Thalmaier, A. (1997). On the differentiation of heat semigroups and Poisson integrals. Stochastics Stochastics Rep. 61, 297-321.

Thalmaier, A. (1998). Some remarks on the heat flow for functions and forms. Electron. Comm. Probab. 3, 43-49.

Thalmaier, A. and Wang, F.-Y. (1998). Gradient estimates for harmonic functions on regular domains in Riemannian manifolds. J. Funct. Anal. 155, 109-124. 